\title{
El particularismo neowittgensteiniano y el rechazo de la teorización en ética ${ }^{1}$
}

\section{Neowittgensteinian particularism and rejection of theorization in ethics}

\author{
OSCAR HORTA \\ Universidade de Santiago de Compostela
}

Recibido: 16-07-2008 Aprobado definitivamente: 19-12-2008

\section{RESUMEN}

El particularismo moral rechaza la pertinencia del recurso a los principios en ética. Una serie de autores de filiación postanalítica neowittgensteiniana han asumido esta posición conducidos por planteamientos antiteóricos, que rechazan el rol evaluativo de la filosofía moral. En este artículo se presenta y examina críticamente esta posición. No se afirma aquí que los antiteóricos incurran necesariamente en posiciones inconsistentes, pero se apunta que su crítica a la teoría moral puede estar descaminada, al descansar en una comprensión incorrecta del carácter de la justificación en ética.

\section{PALABRAS CLAVE}

PARTICULARISMO, GENERALISMO, ANTITEORÍA, JUSTIFICACIÓN MORAL

\section{ABSTRACT}

Moral particularism claims that principles have no use in ethics. A number of authors working in the postanalitic neowittgensteinian tradition have held this position, driven by an antitheoretical viewpoint which rejects the evaluative role of moral philosophy. This paper presents and examines this position from a critical perspective. It does not claim that antitheorists necessarily hold inconsistent positions, but it points out that their critique of moral theory may be misinformed, since it rests on a wrong conception of what justification in ethics is about.

KEYWORDS

\section{PARTICULARISM, GENERALISM, ANTITHEORY, MORAL JUSTIFICATION}

1 Trabajo realizado en el marco del proyecto de investigación FFI2008-06414-C0301/FISO.

C Contrastes. Revista Internacional de Filosofía, vol. XIV (2009), pp. 141-157. ISSN: 1136-4076

Licenciatura de Filosofía, Universidad de Málaga, Facultad de Filosofía y Letras

Campus de Teatinos, E-29071 Málaga (España) 


\section{LA POLÉMICA SOBRE LOS PRINCIPIOS}

EL PARTICULARISMO MORAL ES LA POSICIÓN que sostiene que no hay criterios de carácter general que puedan realmente capturar aquello que en cada circunstancia puede ser moralmente relevante, dada la multiplicidad de factores susceptibles de concurrir en cada caso. ${ }^{2}$ De este modo, rechaza que nuestras decisiones morales hayan de ser resueltas conforme a principios, esto es, prescripciones de carácter general. Sostiene que lo que es preciso para ser un agente moral que sepa como obrar en cada caso no es conocer unos principios pertinentes y el modo de aplicarlos en cada caso. ${ }^{3}$ Un agente moral debe poseer algo distinto a esto, una suerte de «percepción moral» o, como lo ha llamado David McNaughton, «visión moral». ${ }^{4}$ Con un término como este se vendría a denotar la capacidad para saber reconocer, cada vez que nos enfrentamos a una decisión moral, los elementos moralmente significativos a tener en cuenta, y así actuar en consecuencia. El particularismo se opone, así, no al universalismo, ${ }^{5}$ sino al llamado generalismo. Una concepción estricta del particularismo llevaría a rechazar totalmente la pertinencia de cualquier clase de principio. ${ }^{6}$ Una visión menos rígida podría aceptar la utilización de principios únicamente como una

2 El particularismo moral ha sido llamado también en ocasiones 'situacionismo', si bien parece que el término 'particularismo' ha acabado por imponerse en la literatura sobre la cuestión (no se ha de confundir 'situacionismo' en este sentido con el que denota una determinada posición política de corte socialista del mismo nombre, claro está).

3 Por supuesto, por 'principio' no se entiende aquí una máxima en sentido deontológico. El sentido que aquí se da a tal término es mucho más amplio, englobando también cualquier criterio para la toma de decisiones de manera general, aun en el caso de que ello se defienda desde un punto de vista consecuencialista, o incluso en relación a la definición del carácter virtuoso de una acción o un agente. Que debamos obrar en cada caso de manera distinta no implica un rechazo del generalismo, si en cada situación obramos guiados por una idea general común (que puede ser, de hecho, lo que nos lleve a actuar de forma distinta en cada caso). Así, por ejemplo, el utilitarismo del acto es una perspectiva normativa prototípica del generalismo: constituye una posición que nos puede llevar a actuar de forma completamente distinta en cada situación, pero siempre conforme a un mismo principio (maximizar la utilidad en cada caso).

4 D. McNaughton, Moral Vision: An Introduction to Ethics. Oxford: Blackwell, 1988.

5 Más aun, el particularismo no se opone necesariamente a ninguna de las dos distintas acepciones que se han dado al término 'universalismo'. En primer lugar, no niega necesariamente que haya razones que no sean relativas a agentes. En segundo lugar, tampoco rechaza necesariamente que debamos ser imparciales -al menos en ciertos casos.

6 Cabría pensar en alguna suerte de compromiso entre generalismo y particularismo, si se entendiese que este podría desembocar en un pluralismo de criterios. Pero es que incluso sería rechazado por el particularismo: este sostiene que no debemos guiarnos por principios, no que debamos multiplicar estos. Un principio para cada situación no es realmente un principio. 
ayuda o atajo para facilitar ciertas tomas de decisiones de los agentes morales, pero siempre de modo subordinado al juicio (o visión) de estos en cada caso. ${ }^{7}$

\section{EL PARTICULARISMO MORAL ANTITEÓRICO POSTANALÍTICO}

Entre las posiciones particularistas cabe distinguir dos enfoques. En primer lugar, el particularismo ha sido defendido por una serie de autores dentro de la tradición analítica, como han sido fundamentalmente McNaughton y Jonathan Dancy. ${ }^{8}$ Pero ha surgido también una forma de particularismo post-analítico de inspiración neowittgensteiniana que ha llevado su crítica al generalismo mucho más allá, desde posiciones antiteóricas. Este enfoque reivindicaría la que, en opinión de sus defensores, sería una concepción realista del fenómeno moral. Ahora bien, es necesario tener muy presente que con el término 'realismo' no se buscaría aquí denotar, ni mucho menos, la idea de la existencia objetiva de propiedades morales. Muy al contrario, los autores que abrazan esta posición asumen que lo que podríamos llamar propiedades morales no son características de la realidad independientes de nuestras valoraciones. Las circunstancias moralmente relevantes sólo pueden ser determinadas de forma intersubjetiva (y, de hecho, contextual -y, así, variable-). Lo moral sería aquello que conceptualizamos como moral, lo que entendemos como moral, lo que llamamos moral. Se definiría en función del juego de lenguaje dado en este ámbito. El motivo por el que los partidarios de este enfoque lo han tildado de 'realista' parte de asumir que este sería el que apuntaría a la verdadera naturaleza de lo moral una vez libramos nuestra visión de construcciones irreales sobre ello. ${ }^{9}$

7 Lo que un particularista como Dancy sostendría no es que no tengamos razones para actuar que deban guiar nuestras decisiones, sino (conforme a su concepción holista de las razones) que lo que en un caso puede suponer una razón para obrar de un modo determinado, en otro distinto puede ser una razón para actuar de otra forma. Véase J. Dancy, «The Particularist's Progress», en B. Hooker y M. O. Little (eds.), Moral Particularism. Oxford: Clarendon Press, 2000, pp. 130-56.

8 Véase fundamentalmente McNaughton, Moral Vision; J. Dancy, Moral Reasons. Oxford: Blackwell, 1994; Practical Reality. Oxford: Oxford University Press, 2000; y Ethics without Principles. Oxford: Oxford University Press, 2006.

9 En realidad, esta ambigüedad lingüística parte de que el propio término 'realismo moral' no es realmente el más adecuado. Con respecto a la existencia de propiedades morales podemos sostener, al menos, seis posiciones distintas. Cabe entender, por una parte, que estas existen objetivamente y son naturales, que son objetivas y sobrenaturales, o que son objetivas y sui generis. Y, por otra parte, que existen y son subjetivas, que no existen o que su existencia no puede ser conocida. En rigor, las posiciones que se posicionan a favor de la realidad de estas proposiciones son las cuatro primeras. Sin embargo, como es sabido, el término 'realismo moral' solamente denota las tres primeras, dado que no implica sólo realidad sino también objetividad. 
Se rechazaría así, de hecho, lo que en la lingua franca de la metaética ha recibido el nombre de 'realismo moral'. Pero no solamente esto. Los seguidores de esta forma de particularismo vendrían a defender la necesidad de presentar el fenómeno moral de manera libre de las que, en su parecer, serían las distorsiones realizadas sobre este desde la ética normativa. Estas darían lugar a una imagen irreal del fenómeno moral, al pretender dotarlo de una lógica externa a su propia naturaleza.

Así, no es que esta concepción de lo moral niegue que efectivamente haya propiedades morales en el mundo externas a lo que los agentes morales ${ }^{10}$ ven como moralmente significativo, sino que entiende que la pregunta sobre si estas efectivamente existen carece de sentido. Lo moral en nuestras vidas, simplemente, es. No hay nada que investigar más allá en relación a la naturaleza de las circunstancias que vemos como morales. No se rechaza, pues, un determinado resultado al que podríamos llegar al llevar adelante una búsqueda de este tipo, sino tal búsqueda en sí.

De acuerdo con esto, lo que los partidarios de esta perspectiva propondrían sería retornar a la naturaleza de juego de lenguaje de lo moral. Conforme a las premisas wittgensteinianas seguidas por estos autores, toda reflexión que intente probar la falsedad de los sentidos que les damos a las cosas está profundamente desencaminada. El pensamiento moral debería tener como objeto descubrir tales sentidos, no imponer unos nuevos.

El punto de partida para defender esto viene a ser el rechazo wittgensteiniano de la teorización filosófica. Como es sabido, Wittgenstein pondrá de manifiesto que en toda una serie de situaciones desde la filosofía se buscan en vano justificaciones y explicaciones de lo que tenemos ante nosotros. Esto resulta improcedente por el simple motivo de que tales justificaciones no existen, o son simplemente inalcanzables para nosotros. Es por esto, afirma Wittgenstein, que el intento de dar cuenta de por qué hemos de obrar de un modo u otro ha de verse llevado a su fin cuando

he agotado los fundamentos, he llegado a roca dura y mi pala se retuerce. Estoy entonces inclinado a decir: «Así simplemente es como actúo».11

Ahora bien, la idea que está aquí subyaciendo es todavía más radical que la de que no tenemos realmente justificaciones trascendentes para justificar una $u$

10 El propio concepto de «agente moral» no parece ser realmente del agrado de Diamond, en la medida en que implique pensar en un sujeto que lleva a cabo un razonamiento práctico por el que busca justificar sus acciones. Pero puede aceptarse, simplemente, para referir a quien toma decisiones de tipo moral.

11 L. Wittgenstein, Investigaciones Filosóficas. Barcelona: Crítica, 1988, §217. 
otra opción en ética. Lo que se está poniendo aquí realmente de manifiesto es una concepción descriptiva, no normativa, de la filosofía. ${ }^{12}$ Incluso aunque al hablar en términos morales nos refiramos a normas por las que nos guiamos, el planteamiento es no-normativo, puesto que parte del hecho de que nos comportamos de ciertas maneras y entendemos intuitivamente que así hemos de hacerlo como algo dado, rechazando la pertinencia de una reflexión cuya intención sea prescriptiva con respecto a este. Y esta será fundamentalmente la lección del planteamiento wittgensteiniano de la que tomarán nota los antiteóricos actuales en filosofía moral.

Cabe hacer un apunte sobre esto. A menudo se ha supuesto que la aportación de Wittgenstein a la reflexión sobre la moral ha sido relativamente simple, reduciéndose básicamente a una mera limitación de lo que constituye el ámbito de reflexión legítimo que dejaría a esta fuera de juego (su Conferencia sobre ética sería, por supuesto, el lugar comúnmente referido para derivar tal conclusión). ${ }^{13}$ Esto es cierto hasta cierto punto, en la medida en que, como estamos viendo, desde un planteamiento wittgensteiniano se pone coto al alcance de nuestras reflexiones. Pero el hecho es que, cuando se parte ya de las Investigaciones filosóficas, ello se hace únicamente en la medida en que tales reflexiones tengan pretensiones teóricas normativas. No se anula otro tipo de pensamiento en este campo, ${ }^{14} \mathrm{y}$, así, desde premisas wittgensteinianas se llega a una concepción determinada en filosofía moral, que como he venido indicando no es otra que el particularismo.

Pues bien: ¿cómo sucede esto? ¿Cómo se da el paso de la antiteoría al particularismo? Para entender el modo en que esto ocurre hay que partir de constatar la asunción que los partidarios de este enfoque llevan a cabo de que el fenómeno moral existe (conforme a la acepción de 'realismo' arriba indicada). Los agentes morales siguen ciertas normas de conducta. Ante este fenómeno, los teóricos morales intentarían buscar justificaciones, sobre cuya base se podría llevar a cabo, a su vez, una intervención en la propia moral. Ahora bien, si adoptamos un planteamiento antiteórico tal búsqueda deja de tener sentido. Pero ello no implica negar lo obvio, que es que obramos de ciertas maneras (morales). Lo que

12 Puede verse asimismo, Ibid., §109, así como L. Wittgenstein, Zettel. México D. F.: UNAM, 1985, $\$ 313$ y $\S 315$.

13 En la cual, por otra parte, se puede leer en cierto modo un cierto soporte para las tesis particularistas: sería absolutamente significativo y valioso que pudiésemos verdaderamente hallar un fundamento último para nuestra praxis moral. Pero no podemos encontrar tal cosa, y así, en nuestra vida moral, nos hemos de valer sólo con nuestras intuiciones morales, con toda su precariedad: sólo a ellas, pues, nos habríamos de remitir. Véase L. Wittgenstein, Conferencia sobre ética. Con dos comentarios sobre la teoría del valor. Barcelona: Paidós, 1990.

14 Véase M. O. Little, «On Knowing the 'Why': Particularism and Moral Theory», The Hastings Center Report, XXXI (2001), 32-40. 
implica es que el objetivo de nuestra reflexión sobre este debe cambiar. Como he apuntado arriba, debe pasar a ser su comprensión, huyendo de las imposiciones normativas sobre él que pretenderían los teóricos. Una vez dado este paso, se pasa a sostener que nuestras respuestas a los problemas morales a los que nos enfrentamos no se corresponden con reglas de carácter general. Por el contrario, entienden los particularistas, dependen de los casos concretos ante los que nos hallamos en nuestra vida real. Así, intentar englobarlas bajo un patrón que las juzgase conjuntamente implicaría forzar una teorización sobre lo que ya hay y se explica por sí mismo. Resultaría, en línea con lo que se ha apuntado arriba, una forma de violencia sobre el propio contenido de nuestra moralidad. Sería algo artificioso y absurdo, resultado de conceder a la filosofía moral prerrogativas ilegítimas sobre su objeto. ${ }^{15}$ Uno de los partidarios de este enfoque, Carl Elliott, resume las asunciones que subyacen a esta idea como sigue:

[A]sí como es obviamente más fácil entender y aplicar teorías que son claras, económicas, comprehensivas y coherentes, no está claro en absoluto por qué deberíamos esperar que una teoría moral debería ser evaluada en función de tales criterios cuando nuestras propias creencias morales son a menudo genuinamente oscuras, no económicas, no comprehensivas e incoherentes. Para ponerlo de forma muy directa, el conflicto que aquí se presenta se da entre pulcritud y elegancia; queremos que nuestras teorías sean simples y elegantes, pero también ciertas, y la única medida de la «verdad moral» de una teoría parece ser nuestras propias intuiciones, inconsistentes y carentes de pulcritud. ${ }^{16}$

Así las cosas, la teorización sería inadecuada no ya por buscar una justificación que a su vez no estaría justificada, sino por violentar la realidad moral. La complejidad de esta no podría ser acotada bajo patrones generalistas, y ello sería así, en último término, porque rompería las reglas de juego que estos asumirían, al no adecuarse a los requisitos que las construcciones de carácter teórico tendrían que cumplir. Elliott cita en concreto cuatro de estos: simplicidad, economía,

15 Véase a mayores M. O. Little, «Wittgensteinian Lessons on Particularism», en C. Elliott (ed.), Slow Cures and Bad Philosophers: Essays on Wittgenstein, Medicine, and Bioethics. Durham: Duke University Press, 2001, pp. 161-80.

16 «[W]hile it is obviously easier to understand and apply theories that are clear, economical, comprehensive and coherent, it is not at all plain why we should expect a moral theory to measure up to such tests when our own moral beliefs are often genuinely unclear, uneconomical, incomprehensive and incoherent. To put it rather bluntly, the conflict here is one between tidiness and truth; we want our theories to be simple and elegant but also true, and the only measure of the "moral truth" of a theory seems to be our own inconsistent, untidy moral intuitions» (C. Elliott, A Philosophical Disease: Bioethics, Culture and Identity. London: Routledge, 1999, pp. 147-48). 
comprehensividad y consistencia. Y los tres primeros, simplicidad, economía y comprehensividad, son ciertamente criterios cuyo cumplimiento resulta muy conveniente para cualquier teoría. Pero el último, la consistencia (y según el caso también la comprehensividad, si puede ser entendida al margen de la coherencia) parece ser de cumplimiento no ya conveniente, sino imperativo. De este modo, si efectivamente no hay motivo para que la reflexión sobre la moral se guíe por patrones de consistencia, podremos rechazar que la teorización sea necesaria en este campo. Pero no está nada claro que esto pueda ser aceptable. Volveré algo más adelante sobre esta cuestión. Antes, hay algunos otros apuntes que hacer con respecto al carácter de la propuesta neowittgensteiniana en ética.

\section{LA APORTACIÓN DE CORA DiAMOND}

Una de las autoras que más ha destacado entre quienes han abrazado esta posición, y que, de hecho, más ha contribuido a darle forma, ha sido Cora Diamond. Conforme a las premisas arriba referidas, Diamond se opone a la que llama «the orthodox view» en filosofía moral. ${ }^{17} \mathrm{El}$ uso que hace de este término es algo variado. En principio, Diamond lo utiliza en ciertos contextos en los que el objeto de su ataque es la teorización en ética. En línea con lo dicho en el apartado anterior, Diamond critica el hecho de que esta buscaría cuestionar si los conceptos morales que asumimos se encuentran justificados o no. Tal tarea normativa sería inútil y perniciosa, pues sólo puede llevarnos a negar precisamente aquello que dota de sentido a la moral: las propias respuestas morales que de manera directa manifestamos. Ante lo cual podemos preguntarnos, ¿qué espacio deja Diamond para la reflexión sobre la moral? ¿Rechaza esta en su conjunto? Lo cierto es que no. Pero interpreta de un modo muy distinto su rol, en línea con la concepción neowittgensteiniana del papel de la filosofía. Entiende que lo que debería hacer es centrarse en desentrañar el sentido de nuestras afirmaciones morales. Asume, de hecho, que estas constituyen nuestra única referencia ética, sosteniendo que, de hecho, no hay ninguna otra instancia a mayores de ellas desde la que podamos evaluarlas. Diamond parte de las asunciones morales que de hecho tenemos, las cuales adoptamos por pertenecer a una determinada comunidad, en la que nos socializamos y aprendemos el uso de un determinado lenguaje moral. ${ }^{18}$ Así, la filosofía moral ha de ser una reflexión sobre estas que nos lleve a comprender su sentido de

17 C. Diamond, «The Importance of Being Human», en D. Cockburn, David, Human Beings. Cambridge: Royal Institute of Philosophy, 1991, p. 53.

18 Véase Diamond, «The Importance of Being Human», pp. 35-62. 
manera más adecuada. ${ }^{19} \mathrm{Y}$ hay un lugar, también, para el razonamiento moral y la discusión, en la medida en que podamos intentar que otros adopten nuestras convicciones. Para ello, hemos de lograr que nuestras razones lleven a que surjan en aquellos con quienes dialogamos unos contenidos imaginativos como los que en nuestro caso nos llevan a asumir las posiciones que adoptamos. En la concepción de Diamond, aquello en lo que radica la clave de nuestra vida moral lo constituyen estas respuestas imaginativas, que surgen cuando nos enfrentamos a las situaciones a las que se enfrenta la moral. Y para Diamond, puede decirse que las que podemos llamar nuestras formas imaginativas de significado moral son susceptibles de análisis, pero no de análisis crítico normativo. Esto último, en opinión de Diamond implicaría

tratar la reflexión acerca de la moralidad como si esta pudiese ser llevada a cabo sin perdernos en un contexto vaciado de toda familiaridad con tales contenidos imaginativos. $^{20}$

Una posición de este tipo va a ser susceptible de ser cuestionada de varias maneras. En las siguientes secciones expondré algunas de las críticas que se le puede plantear, que, si tienen éxito, socavarán de manera notable el atractivo que un planteamiento antiteórico neowittgensteiniano puede tener. Por supuesto, una de las críticas más fundamentales que se le puede hacer es que no tiene elementos sólidos para cuestionar el que podemos llamar «parroquialismo moral», de manera que cualquier posición discriminatoria o arbitraria podrá resultar perfectamente adecuada conforme a una perspectiva de este tipo. ${ }^{21} \mathrm{Si}$ esta es el resultado de la socialización de los agentes morales en un determinado contexto moral que la asuma, poco vamos a poder oponer a ella (como ha indicado Jeff McMahan). ${ }^{22}$ Asimismo, puede criticarse a esta posición que traiciona su punto

19 Para una aclaración más en detalle de las concepciones de Diamond sobre estas cuestiones pueden verse sus comentarios en C. Diamond, «How Many Legs?», en R. Gaita, (ed.), Value and Understanding: Essays for Peter Winch. London: Routledge, 1990, pp. 149-78; y The Realistic Spirit: Wittgenstein, Philosophy and the Mind. Massachusetts: MIT Press, 1995, en particular los capítulos en este volumen «Anything but Argument?», pp. 291-308; «Eating Meat and Eating People», pp. 319-34; y «Having a Rough Story about What Moral Philosophy Is», pp. 367-81. Véase también C. Diamond, «Injustice and Animals», en Elliott (ed.), Slow Cures and Bad Philosophers, pp. 118-48.

20 «[T]o treat thought about morality as capable of going on without loss in a context emptied of all intimacy with such imaginative shapings» (Ibid., p. 48n).

21 Los intentos de Williams de mostrar que de una posición a favor del parcialismo en ética no se derivan posiciones racistas constituirían un intento de responder a tales críticas (véase B. Williams, Ethics and the Limits of Philosophy. London: Fontana, 1985).

22 J. McMahan, «Our Fellow Creatures», Journal of Ethics, IX (2005), pp. 353-80. 
de vista básicamente interpretativo en la medida en que introduce efectivamente un contenido normativo al prescribir cuál debe ser el modo en el que los filósofos morales deben operar. Aquí, sin embargo, no exploraré estas objeciones. Me centraré en las críticas que cabe plantear a este enfoque a la luz del carácter de los conceptos de consistencia y justificación. Pero antes de pasar a esto es de interés considerar una cuestión más. He presentado aquí la crítica de Diamond a la posición «ortodoxa» entendiendo con este término, básicamente, la teorización con intención normativa en ética. Pero ya indiqué que esta expresión tiene a veces otros sentidos para Diamond. Si analizamos lo que Diamond dice cuando lo emplea, encontramos que en ocasiones define de manera algo más restrictiva lo que nombra con él. Dirá Diamond que la posición «ortodoxa» en filosofía moral se caracterizaría por sostener una ontología moral dividida entre ciertos entes dotados de propiedades moralmente relevantes y las demás entidades que pueblan nuestro mundo. ${ }^{23}$ Lo hace al afirmar que, según esta concepción, «la preocupación por un ser es moral sólo si descansa en propiedades moralmente relevantes». ${ }^{24} \mathrm{Al}$ escribir esto, Diamond parece tener en mente básicamente la teoría moral moderna. De hecho, serían paradigmáticas de su descripción, por una parte, la teoría kantiana (con su hincapié en la consideración de la relevancia de la racionalidad), y, por otra, la utilitarista (que, en cambio, se centraría en la capacidad de ser dañado o beneficiado como lo significativo a la hora de tener en cuenta a alguien). En realidad, esta caracterización resulta cuestionable. La teoría ética aristotélica, por ejemplo, nos remitiría a una forma de comprender nuestras razones para actuar muy distinta, no se adecuaría a este esquema de la cuestión que considera Diamond. Y, sin embargo, qué duda cabe de que efectivamente constituye un claro ejemplo de teoría. Más aun, en la modernidad, y hoy mismo, encontramos también teorías que hacen hincapié asimismo en las relaciones entre el agente y quienes son susceptibles de recibir su atención (por ejemplo, encontramos esta idea en posiciones de inspiración humeana). Este error de Diamond es muy revelador no por sí mismo, sino debido a que pone de manifiesto una visión un tanto simplista de qué es aquello en lo que puede consistir la teorización en ética, que es algo que precisamente pondrán de manifiesto las siguientes secciones.

23 «[C]oncern for a being is moral only if it rests on morally relevant properties» (Diamond, «The Importance of Being Human», p. 53). Véase asimismo Diamond, The Realistic Spirit, p. 322.

24 Ibid. 


\section{El LUGAR DE LA CONSISTENCIA}

La búsqueda de adecuación de nuestros modos de conducta a principios generales puede chocar con las que pueden ser nuestras respuestas imaginativas concretas en determinadas situaciones particulares. Y esto no resultará aceptable para Diamond. En realidad, esta sostendrá que no será realmente posible que podamos vernos motivados para obrar de un modo determinado si no poseemos los contenidos imaginativos necesarios para ello, independientemente de las razones que se nos puedan aportar para hacerlo. ${ }^{25}$ Conforme al planteamiento de Diamond, aquellos que no compartan la idea de que es preciso justificar nuestros juicios morales permanecerán impasibles ante la argumentación que podamos dar en búsqueda de una propuesta que unifique nuestras respuestas ante casos concretos. Así las cosas, ¿qué motivos podríamos tener para realmente exigir tal estabilidad en nuestras respuestas? Cabría pensar que ambas posiciones (la que exige una constancia de nuestras respuestas y la que prefiere nuestras respuestas intuitivas sacrificando esta) se hallarían en igualdad de condiciones: una carece de poder de convicción ante quienes asumen que en ética no cuenta la consistencia entre nuestras decisiones particulares, la otra, ante quienes creen que estas han de resultar consistentes. Se podría indicar que dentro de los límites de la coherencia es posible el debate y el consenso. No obstante, Diamond podría responder que también es posible llegar a tener concepciones imaginativas coincidentes a partir de un diálogo en el que no se busque la depuración de inconsistencias entre las justificaciones que demos para actuar de un determinado modo en cada caso, sino la comunicación de tales concepciones y de los sentidos ligados a ellas.

Cabría sostener que el abandono de la estabilidad en nuestras distintas decisiones morales implica un desentendimiento de la consistencia. Y este tiene unas consecuencias muy serias. Nuestro conocimiento del mundo, más aun, nuestra posibilidad de llevar adelante nuestras vidas en él, sería imposible si no pensásemos de forma consistente. Renunciar a la consistencia implica, pues, un precio carísimo. Más aun, cabría argumentar que alguien, pese a sostener en principio concepciones imaginativas como las de Diamond o Elliott, podría obrar de modo inconsistente con respecto a ellas. No hay modo en que estos antiteóricos puedan argumentar contra esto más que apelando a una forma $\mathrm{u}$ otra de consistencia. ${ }^{26}$

25 Véase sobre esto, en Diamond, The Realistic Spirit, «Anything but Argument?» y «Eating Meat and Eating People».

26 Esta objeción sería formulable en menor grado en el caso de posiciones particularistas menos radicales que las de Diamond, basadas en la complejidad de cada decisión moral, que nieguen la posibilidad de prescribir qué principios cabría hacer valer en cada caso, pero que no abandonen la búsqueda de consistencia en nuestras respuestas. 
Una posición antiteórica puede verse afectada por esta crítica, como mínimo parcialmente. Ello es así dado que, como ya hemos visto, un autor como Elliott explícitamente defiende que nuestras intuiciones morales son inconsistentes, ${ }^{27} \mathrm{lo}$ cual vendría a constituir, dado su esquema conceptual, una defensa de la inconsistencia. Con todo, podría intentarse tal vez mantener un esquema antiteórico sin pretenden acabar con la consistencia en ética. No está claro que ello resulte posible: se puede sostener que estamos ya haciendo teoría, por simple que esta sea, en la medida en que pensamos cómo pueden ser las cosas intentando limar las posibles inconsistencias. Pero, incluso aunque sólo sea en pos del argumento, podríamos dejar de lado esta objeción. Sería posible aceptar entonces que lo que rechazarían los antiteóricos es que nuestras decisiones deban amoldarse a un patrón común. Una respuesta particularista en este punto pasaría por indicar que en cada decisión moral puede haber factores muy diferentes a tener en cuenta. De esta forma, no incurriríamos en una inconsistencia cuando ante casos aparentemente semejantes obramos de forma distinta, simplemente porque tales casos no serían en realidad idénticos. El modo en el que deberíamos obrar en cada caso concreto sería el que proceda en función de las circunstancias que definan este, y ahí acabaría todo. No vendría al caso intentar buscar una lógica subyacente que intente asimilar entre sí las respuestas que demos ante casos distintos, en los que haya factores relevantes diferentes.

Por supuesto, se puede apuntar que en muchas ocasiones tenemos intuiciones contrapuestas. Ante esto, desde el particularismo se podría responder que, de hecho, los dilemas morales forman parte de la vida, y que tampoco las posiciones generalistas escapan a ellos. Pero los generalistas más estrictos, aquellos que estén dispuestos a asumir las consecuencias de su opción por un determinado principio incluso cuando las consecuencias sean contraintuitivas, se ven indemnes ante esta objeción. La apelación a la consistencia constituye, pues, un argumento plausible en contra del particularismo, aun y cuando sea sólo parcialmente. Y, como hemos visto, hay motivos, de todos modos, para considerar que no lo es sólo de este modo parcial.

\section{V. ¿A QUÉ ESTAMOS LLAMANDO JUSTIFICACIÓN EN ÉTICA?}

Por otra parte, habría que apuntar que aun si la acusación de inconsistencia contra los particularistas antiteóricos podría no comprender propiamente qué es aquello que estos realmente defienden, el hecho es que puede que también los propios antiteóricos, a su vez, estén haciendo demasiadas asunciones con

27 Véase sobre esto Elliott, A Philosophical Disease, en concreto el capítulo «A General Antitheory of Bioethics», pp. 141-64. 
respecto a lo que vendrían a sostener las posiciones que rechazan. Como hemos visto, la búsqueda de justificación de nuestras decisiones morales es un objetivo principal de sus ataques. Ahora bien, en este punto no termina de estar claro qué es exactamente lo que quienes defienden posiciones como la de Diamond entienden por 'justificación'. El planteamiento de la idea de justificación de esta autora resulta confuso. Los ejemplos que pone al respecto se refieren a aquellas posiciones que consideran que nuestras actitudes hacia los demás dependen de las capacidades, tomadas por moralmente relevantes, de estos, justificándose así en la medida en que se comprueba que tales facultades son efectivamente poseídas. Hemos visto que Diamond considera típica de la «visión ortodoxa» la idea de que la consideración moral depende de la posesión de ciertas capacidades. Sin embargo, hay ciertamente otros motivos en los que se puede buscar la justificación de una toma de decisión moral, de este tipo o de otro. Sin abandonar un punto de vista neutral, esta puede entenderse en términos de necesidades, por ejemplo. Y más aun, de hecho cabe defender propuestas de justificación de nuestras tomas de decisiones moral centradas en los propios agentes (que a lo que den peso sea a la determinada relación que estos tengan con aquellos o aquellas susceptibles de ser objeto de su consideración).

Sin embargo, en realidad, el procedimiento para la búsqueda de justificación puede ser en sí contrario a los esquemas neowittgensteinianos, pero en el fondo quizás no se aleje tanto como suponen los antiteóricos de la apelación a nuestras motivaciones pre-reflexivas para actuar. El motivo es el siguiente. Lo que en último término comparten los planteamientos teóricos de la justificación moral, es su búsqueda de consistencia entre las prescripciones consideradas justificadas. Los planteamientos fundacionalistas introducen ciertas premisas que el coherentismo no asume, pero aceptan también lo que caracteriza a este, que no es otra cosa que la necesidad de consistencia arriba apuntada. Más aun: es un error notable suponer que el generalismo implica un posicionamiento fundacionalista. Podemos pensar, sin duda, en posiciones generalistas que asuman el esquema fundacionalista (las «pruebas» del imperativo categórico o el principio de utilidad por Kant y Mill serían paradigmáticas en este sentido), ${ }^{28}$ pero el generalismo es igualmente compatible con un planteamiento puramente coherentista (de hecho, lo es también un generalismo no realista moral). Esto se ve con claridad en el caso de los partidarios del método del equilibrio reflexivo. Lo que es más: si vemos qué ocurre en el caso del particularismo, encontramos que una posición analítica particularista holista de las razones como la de Dancy es obviamente coherentista. Pero incluso puede decirse que los antiteóricos neowittgensteinianos podrían ir más allá. Parece que su posición

28 I. Kant, Fundamentación de la metafísica de las costumbres. Barcelona: Ariel, 1996; J. S. Mill, El utilitarismo. Madrid: Alianza, 1984. 
es difícilmente sostenible si no satisface al menos un criterio coherentista. Pero es que, de hecho, en la medida en que parten de nuestras intuiciones morales como una base incuestionable, asumen una posición que en un sentido es ya fundacionalista. Es esencial aquí tener en cuenta que ello es posible en la medida en que puede sostenerse un fundacionalismo no universalista. El hecho de que estemos habituados a que toda una serie de posiciones que apelan a grandes principios universalistas busquen su justificación conforme a una perspectiva fundacionalista puede hacernos olvidar esto. ${ }^{29}$

Por supuesto, de aquí no se infiere que podamos reducir un planteamiento antiteórico a lo que los puntos de vista teóricos habrían asumido tradicionalmente. Pero muestra que las diferencias habidas entre ambos son quizás menos radicales de lo que los antiteóricos neowittgensteinianos habrían asumido, como resultado, posiblemente, de una inadecuada comprensión de lo que desde la teoría moral se asumiría en realidad.

\section{LA BÚSQUEDA DE JUSTIFICACIÓN COMO UNA INCLINACIÓN PRE-REFLEXIVA}

Por otra parte, una interesante objeción que cabe plantear aquí a los antiteóricos radica en que, para la mayoría de nosotros, en una discusión de este tipo sí se podrían dar razones a mayores para encontrar justificadas o injustificadas posiciones como las referidas. Muchos de nosotros encontramos que el hecho de que estas sean consistentes o no con el resto de los puntos de vista que adoptemos es algo relevante para aceptarlas o no. Merece la pena indicar esto particularmente dado que, como hemos visto, Diamond sostiene que la concepción que ella tiene con respecto a nuestras actitudes pre-reflexivas es la que los agentes morales vendrían a sostener (siempre que no hubiesen sido viciados por el estudio de la filosofía moral académica). Y lo mismo sucedería en lo que respecta a la forma de pensar sobre ellas. Sin embargo, este no parece ser el caso. En primer lugar, como es meridianamente claro, aquellos que se adentran en el estudio de la filosofía moral parten de concepciones no necesariamente distintas de las de quienes no lo hacen. En segundo lugar, incluso aunque aceptásemos que los filósofos morales pueden tener una disposición a llevar adelante un escrutinio tal vez más riguroso de sus convicciones morales (lo cual no siempre es cierto), el hecho es que este no es sólo llevado a cabo

29 Frente a Kant y Mill como claros exponentes de esta visión, puede contrastarse la exploración que lleva a cabo Sidgwick ante el «dualismo de la razón práctica». Pese a buscar defender una moralidad universalista, reconoce Sidgwick que el egoísmo ético no es irracional, y puede ser construido siguiendo un modelo fundacionalista (véase H. Sidgwick, The Methods of Ethics. $7^{\mathrm{a}}$ ed. Bristol: Thoemmes Press, 1996). 
por ellos. Más aun, cabe apuntar que la reflexión académica sobre el fenómeno moral no surge de la nada: es llevada a cabo por sujetos que tienen ciertas intuiciones, y que llegan a conclusiones que en último término descansan en estas. Asimismo, la intención de justificar nuestras respuestas morales no es algo exclusivo de quienes tienen una formación académica en filosofía moral, sino que es parte de las concepciones morales de muchos, si no de una gran mayoría, de nosotros. ${ }^{30}$ De hecho, a menudo se asumen (no sólo a nivel puramente individual, sino también de manera extendida dentro de una determinada moralidad) no sólo prescripciones que pueden entrar en conflicto, sino también justificaciones para estas que son contradictorias. Lo que es más, tales justificaciones contradictorias pueden darse incluso en el caso de prescripciones que no entran en conflicto. Frecuentemente creemos en un cierto caso $a$ que debemos actuar de un cierto modo y en otro caso $b$ que debemos obrar de otro, siendo ambos modos para actuar consistentes, pero damos motivos para actuar en $a$ que resultan contradictorios con los dados para hacerlo en $b$. Obsérvese que el particularismo puede perfectamente rechazar que en $a$ y en $b$ debamos actuar del mismo modo, sosteniendo que aquello que es moralmente significativo no tiene por qué ser lo mismo en ambos casos, dado que, a pesar de las similitudes, habría también diferencias (quizás a primera vista imperceptibles) entre estos. De este modo, las razones o motivos para actuar que tendríamos en cada una de estas situaciones serían diferentes. Pero esto es muy distinto de aceptar dos razones contradictorias en $a$ y en $b$, que es algo que el particularismo no puede hacer si quiere mantener la consistencia. Ante esto, por supuesto, la respuesta particularista puede pasar por negar de raíz el problema, al asumir que precisamente lo cuestionable radica en la propia búsqueda de justificación, puesto que esta supondría una ficción externa a nuestra propia vida moral. Asumimos determinadas prescripciones en función de nuestra forma de vida, y punto. Sin embargo, ha de tenerse en cuenta que muy a menudo esta visión particularista no es la que en realidad se asume por los agentes implicados. Lo que estos entienden es que están realmente justificando (o, si se prefiere, queriendo justificar) sus decisiones. Y al hacerlo están dando razones para actuar en cada caso que no son meramente distintas y justificables por la particularidad de cada caso, sino contradictorias entre sí. De este modo, las distintas decisiones morales que dan muchos agentes morales no tienen por qué ser consistentes, puesto que su explicación (por parte de los propios agentes) no se adecuaría a un modelo no ya antiteórico neowittgensteiniano, sino ni siquiera particularista analítico.

30 Asimismo, no hay que asumir que se dé efectivamente una ruptura entre las concepciones morales que quienes han tenido una formación académica sostenían con anterioridad a recibir esta y la que sostienen con posterioridad, sino que en muchos casos lo que hay es un continuo entre ambas. 
Y cabe añadir algo más en este punto. La denuncia de la búsqueda de justificaciones se da sobre la base de que estas, supuestamente, dejarían de lado las inclinaciones pre-reflexivas que tenemos, que serían lo realmente significativo en términos de motivación moral. Sin embargo, tal acusación deja de lado algo importante. Podemos sostener perfectamente que tales inclinaciones pre-reflexivas son lo que hemos de tener en cuenta para guiar nuestra acción moral, pero buscar la consistencia allí donde estas choquen. Obraríamos así precisamente para hacer valer aquellas inclinaciones que consideramos más significativas. Más aun, es importante tener en cuenta que la propia búsqueda de consistencia puede ser considerada una inclinación previa a la propia reflexión, no ya en un nivel lógico (en tanto que constituye un requisito para esta), sino en la medida en que tenemos una fortísima tendencia a buscar que nuestra visión de las cosas y nuestras acciones sigan patrones consistentes ${ }^{31}$ (lo cual no es extraño, dada su utilidad para nuestra supervivencia). La crítica de esta explicación de nuestra búsqueda de justificación de nuestras acciones podría resultar válida si aceptásemos una teoría que rechace la explicación que acabamos de dar, esto es, si asumiésemos una teoría racionalista de la motivación moral ${ }^{32} \mathrm{de}$ forma ligada a la asunción del externismo de las razones para actuar. ${ }^{33}$ Como es sabido, según la primera, entenderíamos que aquello que nos motiva a actuar son razones (en lugar de deseos); según el primero, nuestros motivos para actuar son objetivos, de manera que podemos tenerlos aun en el caso de que ni los reconozcamos como tales ni se encuentren conectados con motivos que reconozcamos como tales. Asumiendo esto, resulta claro que rechazaríamos la idea de que la búsqueda de consistencia es algo que llevemos adelante porque tengamos alguna clase de deseo, o porque queramos armonizar ciertas preferencias subjetivas que tengamos que puedan chocar. Ahora bien, precisamente tal concepción intro-

31 Véase sobre esto I. Persson, The Retreat of Reason: A Dilemma in the Philosophy of Life. Oxford: Oxford University Press, 2005, p. 4.

32 Hay una amplia bibliografía sobre esta cuestión. Una posición clásica a favor de una teoría de la motivación de corte racionalista es la defendida en T. Nagel, The Possibility of Altruism. Princeton: Princeton University Press, 1970. Una posición representativa a favor de una posición contraria, de carácter volitivista, es la sostenida a su vez en M. Smith, «The Humean Theory of Motivation», Mind, XCVI (1987), pp. 36-61. Clásicamente se asumen estas posiciones, respectivamente, a Kant y a Hume, si bien no está claro que la posición de este último se adecue con rigor a lo que los planteamientos volitivistas mantendrían.

33 Textos básicos con respecto a esta cuestión son B. Williams, «Internal and External Reasons», en su Moral Luck: Philosophical Papers 1973-1980. Cambridge: Cambridge University Press, 1981, pp. 101-13; J. McDowell, «Might There Be External Reasons», en J. E. J. Altham y R. Harrison (eds.), World, Mind, and Ethics: Essays on the Ethical Philosophy of Bernard Williams. Cambridge: Cambridge University Press, 1995, pp. 68-85; o D. Parfit, «Reasons and Motivation», Supplement to the Proceedings of The Aristotelian Society, LXXI (1997), pp. 99-130. 
duce móviles morales que no son, ciertamente, pre-reflexivos. Con ello, se nos proporciona una explicación teórica del fenómeno moral con la que estarán de acuerdo particularistas analíticos como Dancy, ${ }^{34}$ pero que introduce un modo de reflexión en filosofía moral que resulta ajeno a una concepción neowittgensteiniana. Esto es así en la medida en que, como podemos ver, de operar conforme a este estaríamos buscando justificaciones más allá del sentido que en nuestra vida diaria daríamos a los conceptos morales que utilizamos.

\section{RECAPITULACIÓN Y CONCLUSIÓN}

En este artículo se ha intentado dar cuenta del punto de vista particularista antiteórico, mediante el examen de la crítica que desde este se ha efectuado a la teorización y la búsqueda de principios en ética. Cabe establecer dos planos en los que tal crítica ha tenido lugar, uno normativo y otro metaético. El primero de estos se concretaría en la oposición al generalismo. Sería, pues, donde se manifestaría propiamente lo que de particularista tiene esta posición. Hemos visto que, pese a lo que se podría pensar a primera vista, el rechazo de los principios no resulta necesariamente inconsistente (aunque, por supuesto, puede serlo). Quienes defiendan el particularismo pueden sostener que en cada caso concreto nos encontramos ante situaciones que requieren respuestas particulares, de forma que no es inconsistente actuar de modos distintos ante ellas. Ahora bien, las circunstancias relevantes que el o la particularista capta en cada situación pueden repetirse. De este modo, no está claro por qué el particularismo no puede ser reducido a un generalismo pluralista.

En el plano metaético se manifestaría en especial la otra componente de la posición que aquí ha sido considerada, la antiteórica. ${ }^{35}$ Ello sucedería con el rechazo de la búsqueda de justificación en ética. El particularismo antiteórico defiende que no hay nada que pueda justificar nuestras respuestas imaginativas concretas más allá de estas mismas. Así, la reflexión sobre la moral no debería tener como objeto diferenciar las acciones moralmente justificadas de las que no lo están. Ahora bien, tal y como he intentado mostrar en las secciones precedentes, la búsqueda de la justificación de nuestras posiciones en ética no tiene por qué distanciarse demasiado de tal posición, por extraño que ello parezca a primera vista. Ello es así al menos en la medida en la que se sostenga que el

34 Véanse las obras ya citadas arriba de Dancy, Moral Reasons, Practical Reality y Ethics without Principles.

35 Por supuesto, no hay que considerar la dimensión normativa y la metaética del particularismo antiteórico como compartimentos estancos: aquella es asumida precisamente de la mano de la adopción de esta última. 
abandono de una respuesta imaginativa ha de darse sólo en tanto que choca con otra. Así, lo único que tal vez se introduce más allá de tales respuestas es la búsqueda de consistencia, que parece un requisito francamente aceptable. Más aun, hemos visto que incluso la búsqueda de consistencia puede ser vista ella misma como una respuesta intuitiva concreta que de hecho manifestamos (pues queremos actuar de forma consistente). Pero, si esto es así, ello significa que las posiciones teóricas no se distancian tanto como en principio parecía de las particularistas antiteóricas. ${ }^{36} \mathrm{~A}$ la luz de esto, tenemos motivos para cuestionar la validez de las críticas antiteóricas postanalíticas a la teoría moral. De hecho, tal cuestionamiento puede tener lugar incluso aunque no se rechace el punto de vista particularista. El motivo radica en que la crítica llevada a cabo desde el particularismo antiteórico hace aguas cuando comprobamos que no hay una distinción tan fundamental entre las posiciones que este asume y las que rechaza.

Sólo hay un modo en el que esta conclusión puede ser evitada: si entendemos que efectivamente la posición neowittgensteiniana acepta la inconsistencia. Si es así, no cabe duda de que vamos a encontrarnos ante una diferencia crucial real entre esta perspectiva teórica y la antiteórica. Pero, en ese caso, tendremos motivos para rechazar la crítica que esta hace a la teoría moral, en la medida en que asumamos que la consistencia es un requisito irrenunciable.

Oscar Horta. Universidade de Santiago de Compostela.

Linea de investigación:

Filosofía moral actual, ética aplicada y bioética.

Publicaciones recientes:

«El igualitarismo y la doble confusión de Sidgwick», Télos, 16, 1, 2008, 43-51.

La Filosofía Moral de J. Ferrater Mora, Documenta Universitaria: Girona, próxima publicación.

Dirección postal:

Facultad de Filosofía, Pza. de Mazarelos s/n, 15782 Santiago de Compostela (A Coruña).

Dirección electrónica: Oscar.Horta@usc.es

36 De hecho, estaríamos en todo caso ante una diferenciación que, como mucho, resultaría procedente trazar en el caso del particularismo postanalítico, no en el del analítico. 